\title{
Cows' milk sensitive enteropathy in cystic fibrosis
}

\author{
S M HILL, A D PHILliPS, M MEARNS, AND J A WALKER-SMITH \\ Academic Department of Child Health, Queen Elizabeth Hospital for Children, London
}

SUMmary Proximal small intestinal mucosal biopsies were carried out in children with cystic fibrosis who had diarrhoea and failed to thrive in spite of adequate treatment, including pancreatic supplements. Histological examination of eight of the 17 biopsies taken over a period of 12 years showed evidence of enteropathy, and accounted for one in $13(8 \%)$ children with cystic fibrosis under 3 years of age attending our clinic. Seven responded to a cows' milk free diet; the diarrhoea stopped and weight gain increased. One of these responded only when gluten was also excluded from his diet. The eighth child remained on a normal diet and his symptoms did not improve. The enteropathy had resolved in all five patients who had further biopsies taken while receiving treatment, and from 15 months to 3 years of age all the children tolerated a normal diet and continued to thrive.

Cows' milk sensitive enteropathy is an important cause of failure to thrive in children with cystic fibrosis. Small intestinal biopsy is an important investigation in younger children who fail to thrive and have diarrhoea despite adequate treatment.

Children with cystic fibrosis often fail to thrive. This failure is usually related to the disease itself, and may be the result of recurrent chest infections, inadequate food, or pancreatic enzyme deficiency. Two gastrointestinal diseases that may, in association with cystic fibrosis, cause failure to thrive are coeliac disease ${ }^{12}$ and lactase deficiency. ${ }^{34}$

It is important to make the correct diagnosis when a child with cystic fibrosis is failing to thrive as the severity of the respiratory component of the disease (which may affect prognosis) seems to be related to the child's nutritional state. ${ }^{5}$ The aim of this study was to investigate whether cows' milk sensitive enteropathy is a cause of chronic diarrhoea and failure to thrive in children with cystic fibrosis.

\section{Patients and methods}

Seventeen children were included in the study from 1975-1987. Fifteen of the patients were already attending the cystic fibrosis clinic and were selected for small intestinal mucosal biopsy because they were failing to thrive in spite of adequate treatment for cystic fibrosis. This included pancreatic enzyme and fat soluble vitamin supplements. Two other children underwent small intestinal mucosal biopsy and sweat tests during the same admission as part of the routine procedure for investigation of failure to thrive, and had positive sweat tests. They were taking no medication at the time of biopsy. The weights of all the children were below the 25th percentile, and five were well below the third.

Nine of the children were boys and eight girls. They were aged from 3 months to 12 years (median 12 months). In all cases the diagnosis of cystic fibrosis was made from two positive sweat tests and typical symptoms. Sweat tests were considered positive if at least $100 \mathrm{mg}$ of sweat was collected that contained at least $70 \mathrm{mmol} / \mathrm{l}$ sodium.

The diagnosis of cows' milk sensitive enteropathy was made when characteristic histological findings were present in a proximal small intestinal mucosal biopsy specimen, ${ }^{6}$ and there was a rapid clinical response to the exclusion of cows' milk from the diet. ${ }^{7}$ Early challenge with cows' milk and further small intestinal biopsies were necessary to prove that the disease was present. In practice, as the condition was a temporary one and there was a risk of anaphylaxis on the reintroduction of cows' milk, challenge was not considered necessary if the histology was characteristic and the clinical improvement rapid.

At the time of small intestinal mucosal biopsy, 16 of the children were on cows' milk based feeds, and one was breast fed. The mother of the latter child included cows' milk in her diet. Three children under 6 months old were on full fat adapted formula feeds, three were taking 'cystic mix' (SMA and 
Marvel with added Caloreen), and the other 10 were having skimmed milk. The 13 oldest children included a normal amount of gluten in their diets, but the four youngest had not started solids. Stool samples were taken from all patients and examined for pathogens. The small intestinal mucosal biospy specimens were taken after full informed consent had been given by the parents.

After an overnight fast, a paediatric sized double port capsule was used to obtain mucosa from the duodenojejunal flexure under radiological control. In addition to the biopsy specimen, duodenal juice was aspirated if possible and examined for the presence of Giardia lamblia. A sample of tissue from one biopsy specimen from each patient was frozen in liquid nitrogen and used to assay disaccharidases ${ }^{8}$ and the other was fixed in formal saline and processed for histological examination. Suitable specimens were also analysed morphometrically using a computer based image analysis system. ${ }^{6}$ If the histological appearance of a biopsy was abnormal and consistent with a cows' milk sensitive enteropathy ${ }^{6}$ the child was given a cows' milk exclusion diet for the next few months, usually until at least 15 to 18 months of age. These children were reviewed regularly in the outpatient clinic with particular attention being paid to weight gain and stool frequency. If possible proximal small intestinal mucosal biopsy was repeated before cows' milk was reintroduced to their diets. Morphometric analysis of the biopsy specimens was again performed and the results were compared with those of the specimens obtained on presentation.

Other features of the children with enteropathies that were reviewed were: the manner of initial presentation with cystic fibrosis; a personal or family history of atopy; and any investigations which might have indicated an atopic tendency. These included examination of peripheral blood for eosinophils, skin prick tests for allergens, and estimation of serum IgE and specific IgE concentrations. Sixteen per cent of all children attending our clinic during the 12 year period presented with meconium ileus.

\section{Results}

In nine of the 17 children proximal small intestinal biopsy specimens were histologically normal. They were aged from 3 months to 12 years (median 2 years). Seven were girls and two boys. No pathogenic organisms were detected in their stool samples, and $G$ lamblia was not detected in any duodenal aspirate. No gastrointestinal cause other than cystic fibrosis was found to explain their failure to thrive and chronic diarrhoea.
Histological and morphometric findings in the biopsy specimens from the remaining eight children were abnormal and consistent with cows' milk sensitive enteropathy - that is, they had thin mucosa with reduced villous height (fig 1). Seven of these children were boys, and their ages ranged from 5 months to 28 months (median 10). This group included one of the children who underwent small intestinal mucosal biopsy at the same time as cystic fibrosis was diagnosed. Rotavirus was detected by electron microscopy both in the stool and within the small intestinal mucosa of one child. No pathogens were detected in stool or duodenal aspirate from any other patient.

The child with most frequent diarrhoea (10 times a day) had an enteropathy, while the child without diarrhoea did not. Apart from these two cases, there was no obvious difference in stool output between those children with enteropathy and those with normal small intestinal mucosa.

Patients with enteropathy were on a variety of cows' milk based feeds. Three were on formula feeds, two were on cystic mix, one was on doorstep milk, and the other two on skimmed milk.

Four of the eight children with enteropathy had originally presented with meconium ileus, whereas about $16 \%$ of all children attending the clinic over the 12 years had presented in this manner. The other four had presented with failure to thrive, and one also had recurrent chest infections. At the time of small intestinal mucosal biopsy the weights of five were below the third percentile (and the other three on or below the 10th), all their birth weights had

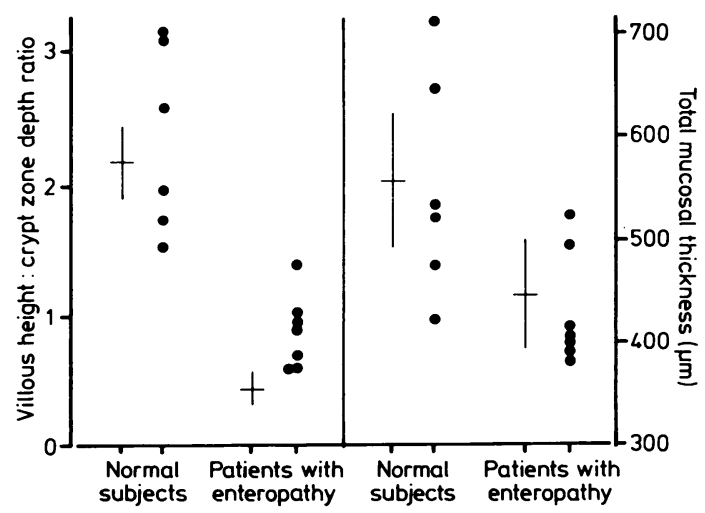

Fig 1 Morphometric findings in patients with and without cows' milk sensitive enteropathy. Dots represent the 13 patients studied, and vertical bars the findings of Maluenda et al (mean $(S D)) .^{6}$ 
been in the normal range. All seven available for follow up had developed atopic symptoms, which included asthma in five, eczema in two, and urticaria in one. Three children had appreciable eosinophilia (more than $400 \times 10^{6} / 1$ ) on at least one occasion.

Disaccharidase activities were normal in the four histologically normal biopsy specimens in which they were measured. Sucrase and maltase activities were normal in the six children with enteropathy in which they were measured. Lactase activity was reduced in two $(0.5$ and $0.8 \mathrm{mmol} / \mathrm{min} / \mathrm{g})$. This was not associated with clinical lactose intolerance in either child.

Seven of the eight children with small bowel mucosal abnormalities compatible with the diagnosis of cows' milk sensitive enteropathy had cows' milk withdrawn from their diets; Pregestimil was used as a substitute feed. The diagnosis does not seem to have been considered in one child who was biopsied in 1976, who remained on cows' milk, and who continued to fail to thrive. He died from a chest infection aged 3 years.

In six of the seven children stool consistency improved, stool frequency fell to two or three times a day, and they had 'catch up' weight gain. One child whose weight on presentation was well below the third percentile $(3.6 \mathrm{~kg}$ at 6 months) almost reached this percentile in six months. One child's weight climbed from the third to the 10th percentile, two from the tenth to the 25th, and two reached the 50 th percentile (one from the third and the other from the 10th).

The one child who did not improve rapidly underwent a further proximal small intestinal biopsy, which showed that enteropathy was still present. Gluten was excluded from his diet in addition to cows' milk, and a third biopsy showed improvement in the histological picture. He underwent two further biopsies, both of which were normal. The first was at 3 years of age after cows' milk was reintroduced to his diet, and the other at 4 years of age when his diet also included gluten, so he had transient gluten intolerance rather than coelic disease.

Small intestinal biopsy was also repeated in four of the seven other children with enteropathy 2-14 months later while they were receiving cows' milk free diets. Reasons for not repeating the biopsy in the other three were: adverse social circumstances $(n=1)$, follow up continued elsewhere $(n=1)$, and cows' milk sensitive enteropathy not recognised $(n=1)$. All five biopsy specimens showed obvious improvement on histological examination.

Four of the five specimens were suitable for morphometric analysis. Fig 2 shows the results of morphometry in these, and compares them with the

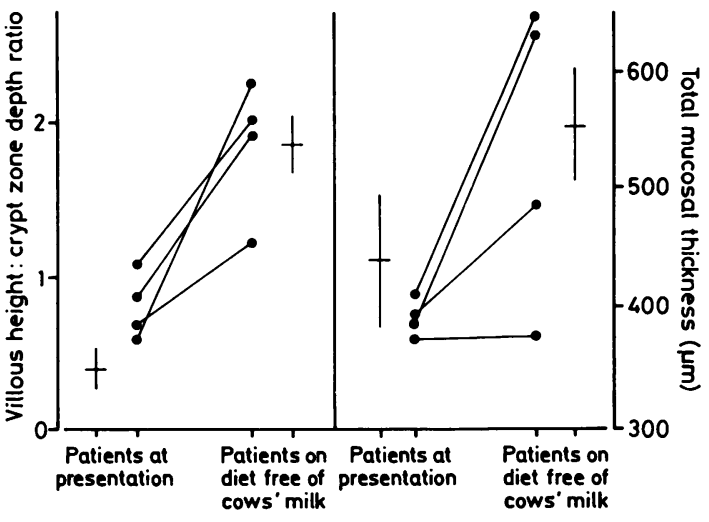

Fig 2 Morphometric findings in patients with cows' milk sensitive enteropathy on presentation and on a diet free of cows' milk. Dots represent the four patients biopsied twice, and vertical bars the findings of Maluenda et al (mean (SD)). ${ }^{6}$

morphometric findings on presentation. In four the mucosal thickness improved, and in all cases the ratio of villous height:crypt zone depth improved.

Disaccharidase activities were again measured, and the child who had had the lowest lactase activity now had one within the normal range $(1 \cdot 8)$.

The children were challenged with cows' milk from 2-13 months (median 10 months) after excluding it from their diets. There were no adverse reactions, weight gain continued at the same rate as previously, and stool frequency was unchanged.

\section{Discussion}

Cows' milk sensitive enteropathy was a common problem among the children under 3 years old $(8 / 13$, $62 \%$ ) who were selected from our cystic fibrosis clinic for small intestinal mucosal biopsy. As 105 children had presented to our clinic by 3 years of age from 1975-87, the overall incidence of the enteropathy in children presenting with cystic fibrosis in this age group was $1 / 13$. The incidence of cows' milk sensitive enteropathy in the general population is difficult to establish, particularly as so many children are started on restricted diets without seeing a doctor, but it seems to be considerably lower than this. ${ }^{9}$

Three possible reasons for a high incidence in children with cystic fibrosis are: the association of cystic fibrosis with atopy ${ }^{10}$ (cows' milk sensitive enteropathy is an atopic related disorder), increased intestinal permeability, ${ }^{11}$ and impaired digestion of protein due to insufficient pancreatic trypsin leading to a high antigen load presenting to the small intestinal mucosa. 
The main features of this group of patients were the unequal sex ratio, the high incidence of atopy, and the presentation with meconium ileus. A predominence of boys with a family history of atopy has previously been reported in a group of children presenting with cows' milk sensitivity. ${ }^{12}$ Atopic symptoms are common in children with cows' milk sensitive enteropathy alone ${ }^{13}$ and as already mentioned atopy is associated with cystic fibrosis. ${ }^{10}$ The incidence of meconium ileus would of course be higher in this group than among all children with cystic fibrosis, as cows' milk sensitive enteropathy is a disease of infancy, and many children with cystic fibrosis present later. The incidence of $50 \%$ is surprisingly high, however, when the overall incidence in our clinic was $16 \%$.

In some cases, although the mucosal appearance had improved when cows' milk was excluded from the diet, it was not entirely normal. This is similar to previous findings ${ }^{6}$ and could indicate that in spite of advice from a dietitian these children may have continued to ingest some cows' milk. Alternatively they may have reacted to Pregestimil or other dietary constituents, although this was not clinically apparent. One child was intolerant of gluten, which illustrates the fact that multiple food intolerances may co-exist. ${ }^{14}$ It was because of this that Pregestimil was used as a cows' milk substitute (rather than a soya based milk), because at least $15 \%$ of children intolerant of cows' milk also react to soya milk. ${ }^{7}$ As all the children treated for cows' milk sensitive enteropathy were given Pregestimil feeds, there were no 'control' children. It could be argued that children with cystic fibrosis, even without cows' milk sensitive enteropathy, would thrive better on Pregestimil than cows' milk feeds because Pregestimil contains fat in the form of medium chain triglycerides which are water miscible and more rapidly hydrolysed by pancreatic lipase than long chain triglycerides. Cows' milk feeds, however, do have the advantages of higher salt and protein contents. When our group of children were restarted on diets containing cows' milk there were no noticeable changes in the rate of weight gain, so there was no evidence that either milk was superior once tolerance to cows' milk had been achieved.

Although cases of cows' milk sensitive enteropathy in patients with cystic fibrosis do not seem to have been reported previously, the enteropathy may have been described. ${ }^{4} 1516$ Antonowicz et al reported 27 cases of cystic fibrosis with steatorrhoea in association with small bowel enteropathy; no explanation for the enteropathies was given. ${ }^{4}$ Reduction in disaccharidase activities was thought to be secondary to the partial villous atrophy (as in our two cases with low activities of lactase on presenta- tion). Nordio et al also described a child with reduced disaccharidases 'associated with a flatness of intestinal mucosa'. ${ }^{15}$ of 17 children with cystic fibrosis described by Gibbons, the one patient with clinical and biochemical evidence of lactase intolerance underwent small intestinal biopsy. ${ }^{16}$ There was flattening of the mucosa with villous atrophy and reduced disaccharidase activity. No explanation was given for the enteropathy. In view of our findings, it seems likely that some of these cases were of cows' milk sensitive enteropathy.

Only two of our children had low lactase activity on presentation, and there was no evidence of lactose intolerance. Lactase deficiency has been associated with cystic fibrosis in the past. ${ }^{3} 41516$ There was a single case report of lactase deficiency in a 3 year old boy in $1963,{ }^{3}$ and three cases were reported in $1966 .{ }^{15}$ In 1968 seven cases were described $^{4}$ and a genetic association with cystic fibrosis was postulated, but on review 10 years later ${ }^{17}$ the incidence was considered to be no higher than in the general population and was thus thought not to be secondary to cystic fibrosis. Other reports have been inadequate as either the diagnosis of cystic fibrosis was in doubt as sweat tests were normal, ${ }^{18}$ or a lactose load did not cause diarrhoea. ${ }^{16}$

In conclusion, cows' milk sensitive enteropathy should be considered in the differential diagnosis of persistent failure to thrive in younger children with cystic fibrosis, particularly in view of our high incidence of $1 / 13$ in patients under 3 years of age. We would not recommend a cows' milk free diet for newly diagnosed infants with cystic fibrosis if they are gaining weight well and have formed stools on treatment with pancreatic supplements, but there may be a place for giving a medium chain triglyceride containing protein hydrolysate formula feed plus pancreatic supplements to newly diagnosed infants who have frequent stools and poor weight gain on treatment with a suitable cows' milk based feed and pancreatic supplements. Some clinicians, however, might prefer to do a small intestinal biopsy first to exclude the rare case of coeliac disease. It is important to make the diagnosis and treat correctly as optimal nutritional state is thought to have such a profound effect on overall health, and possibly survival. ${ }^{19} 20$ Recurrent respiratory infections, which can result in reduced life expectancy, are thought to be less of a problem in a child in a good nutritional state, ${ }^{5}$ and this study shows that cows' milk elimination has an appreciable impact on the nutritional state of some patients with cystic fibrosis.

References
1 Anderson DH. Cystic fibrosis of the pancreas and its relation to 
coeliac disease. A clinical pathology study. Am J Dis Child 1938;56:344-99.

2 Taylor B, Sokol G. Cystic fibrosis and coeliac disease: report of two cases. Arch Dis Child 1973;48:692-6.

3 Cozetto FJ. Intestinal lactase deficiency in a patient with cystic fibrosis: report of a case with enzyme assay. Pediatrics 1963;32: 633-6.

4 Antonowicz I, Reddy V, Khaw KT, Shwachman H. Lactase deficiency in patients with cystic fibrosis. $J$ Pediatr 1968;42: 492-500.

5 Sheppard R, Cooksley WGE, Cook WDD. Improved growth and clinical nutrition and respiratory changes in response to nutritional therapy in cystic fibrosis. J Pediatr 1980;97:351-7.

${ }^{6}$ Maluenda C, Phillips AD, Briddon A, Walker-Smith JA. Quantitative analysis of small intestinal mucosa in cows' milk sensitive enteropathy. J Pediatr Gastroenterol Nutr 1984;3: 349-56.

7 Walker-Smith JA. Milk intolerance in children. Clin Allergy 1986;16:183-90.

8 Phillips AD, Avigad S, Sacks J, Rice SJ, Walker-Smith JA. Microvillous surface area in secondary disaccharidase deficiency. Gut 1980;21:44-8.

9 Rona RJ, Chinn S. Parents' perception of food intolerance in primary school children. $\mathrm{Br}$ Med J 1987;294:863-6.

10 Hodson ME, Warner JO. Immunology. In: Hodson ME, Norman AP, Batten JC, eds. Cystic fibrosis. London: Ballière Tindall, 1983:91-6.

${ }^{11}$ Leclercq-Foucart J, Forget PP, Van Cutsem JL. Lactuloserhamnose intestinal permeability in children with cystic fibrosis. J Pediatr Gastroenterol Nutr 1987;6:66-70.

12 Halpem SR, Sellars WA, Johnson RB, Anderson DW, Sapenstein S, Reisch JS. Development of allergy in children fed breast, soy or cows' milk in the first six months of life. J Allergy Clin Immunol 1973;51:139-51.

13 Digeon B, Walker-Smith JA. Food intolerance and gastrointestinal disease in infancy: personal practice. Dig Dis 1986;4: $139-46$.

14 Vitoria JC, Camarero C, Cojo A, Ruiz A, Rodrigukez-Soriano J. Enteropathy related to fish, rice and chicken. Arch Dis Child 1982;57:44-8.

15 Nordio S, Lamedica GM, Beno A, Vignolo L. Disaccharidase activities of duodenal mucosa in children. Ann Pediatr 1966;206: 287-312.

16 Gibbons ISE. Disaccharidases and cystic fibrosis of the pancreas. Arch Dis Child 1969;44:63-8.

17 Antonowicz I, Lebenthal E, Shwachman H. Disaccharidase activities in small intestinal mucosa in patients with cystic fibrosis. J Pediatr 1978;92:214-9.

18 Jones RHT. Disaccharide intolerance and mucoviscidosis. Lancet 1964;ii:120-1.

19 Goodchild M. Practical management of nutrition and gastrointestinal tract in cystic fibrosis. $J$ R Soc Med 1986;79(suppl 12): 32-5.

${ }^{20}$ Levy LD, Durie PR, Pencharz PB, Corey L. Effects of longterm nutritional rehabilitation on body composition and clinical status in malnourished children and adolescents with cystic fibrosis. J Pediatr 1985;107:225-30.

Correspondence to Dr SM Hill, Department of Child Health, Institute of Child Health, 30 Guilford Street, London WC1N 1EH.

Accepted 8 March 1989 